\title{
A Four-Dimensional Hyperchaotic Finance System and Its Control Problems
}

\author{
Lin Cao \\ School of Business and Administration, Qilu University of Technology, Jinan 250353, China \\ Correspondence should be addressed to Lin Cao; caolinqlu@163.com
}

Received 29 August 2017; Revised 11 December 2017; Accepted 3 January 2018; Published 1 February 2018

Academic Editor: Sundarapandian Vaidyanathan

Copyright (C) 2018 Lin Cao. This is an open access article distributed under the Creative Commons Attribution License, which permits unrestricted use, distribution, and reproduction in any medium, provided the original work is properly cited.

The construction and several control problems of a new hyperchaotic finance system are investigated in this paper. Firstly, a new four-dimensional hyperchaotic finance system is introduced, based on which a new hyperchaos is then generated by setting parameters. And the qualitative analysis is numerically studied to confirm the dynamical processes, for example, the bifurcation diagram, Poincaré sections, Lyapunov exponents, and phase portraits. Interestingly, the obtained results show that this new system can display complex characteristics: chaotic, hyperchaotic, and quasiperiodic phenomena occur alternately versus parameters. Secondly, three single input adaptive controllers are designed to realize the control problems of such system: stabilization, synchronization, and coexistence of antisynchronization and complete synchronization, respectively. It is noted that the designed controllers are simpler than the existing ones. Finally, numerical simulations are provided to demonstrate the validity and the effectiveness of the proposed theoretical results.

\section{Introduction}

It is well known that the classical chaotic attractor was firstly found by Lorenz in 1963 [1]. As a most significant system in nonlinear dynamical systems, chaos systems and their relative problems have attracted a lot of consideration from all kinds of researchers in many fields of science, and many significant results have been obtained in the past few decades; for details, see [2-6] and the references therein. From then on, chaos becomes a hot topic for a broad class of applications in physics, electrical engineering, secure communication, and many other fields [4-6]. As we know that the positive Lyapunov exponent of the chaotic systems is a critical prerequisite in these applications. The threedimensional chaotic system only has one positive Lyapunov exponent; thus it is typically difficult to meet some certain requirements. For instance, the messages which are masked by the simple chaotic systems are easy to decipher in secure communication $[4,5]$. Different from the ordinary chaos, the hyperchaotic system has at least two positive Lyapunov exponents and thus has more prominent advantages due to its higher dimensions and more unpredictable behaviors. Accordingly, the hyperchaotic systems have been investigated extensively, and various control techniques and approaches have been developed and utilized [7-9]. It should be pointed out that the above-mentioned works are based on the hyperchaotic models with complex structure. However, such models are difficult to be verified in reality. This motivates the author to construct a simple system that can display rich dynamical characteristics.

It is well known that the OGY method [10] for chaos control was firstly observed in 1990. Meanwhile, the PC method [11] for chaos synchronization firstly achieved chaos synchronization in 1990. Since then, a lot of researchers who are from all kinds of scientific fields had to pay an increasing interest to investigate chaos systems and their relative problems in the past years, and many significant theoretical results and experiments conclusions have been published; for details please see [12-27] and the references therein. In recent years, several typical kinds of chaos synchronization have been identified, for example, complete synchronization (or synchronization) $[14,15]$, phase synchronization $[16,17]$, lag synchronization $[18,19]$, generalized synchronization $[20$, $21]$, antisynchronization [22, 23], projective synchronization $[24,25]$, coexistence of antisynchronization and complete synchronization $[26,27]$, and simultaneous synchronization 
and antisynchronization [27]. However, for the chaotic or hyperchaotic systems, there are still some important questions needed to be further investigated, such as the complexity of the controllers and the existence of some control problems, which partly motivates the present work.

For the economic systems, the chaotic behavior in those systems was first found in 1985 [28]. It is noted that chaotic behavior in economics implies that the considered economic system has an inherent indefiniteness. Thus, it is of important value to study the chaotic finance system in order to achieve a stable economic growth. A novel chaotic finance system [3] was presented in 2001. There are four subblocks which construct the system model, that is, money, production, labor force, and stock. This system model is described by three state variables of the time variations: $x_{1}$ stands for the interest rate; $x_{2}$ and $x_{3}$ are the investment demand and the price index, respectively. Since this chaotic finance system is proposed, many works have been done for this finance system $[29,30]$. Recently, a novel hyperchaotic finance system [31] is presented in 2010 and some important results have been obtained. However, there are some limitations in the existing results. For example, in the stabilization problem and the complete synchronization problem of such system, the designed controllers are too complicated to be used in applications. On the other hand, the coexistence of antisynchronization and complete synchronization problem of this hyperchaotic finance system has not been investigated so far. Therefore, this new hyperchaotic finance system needs to be further investigated.

Motivated by the above discussions, a four-dimensional hyperchaotic finance system is presented, which can generate double-wing chaotic and hyperchaotic attractors with three equilibrium points. In comparison with the most existing results, this model has simple structure and can display complex dynamics: chaotic, hyperchaotic, and quasiperiodic phenomena occur alternately. Furthermore, some basic dynamic properties of the new hyperchaotic finance system regarding equilibria, dissipation, Lyapunov exponent, Lyapunov dimension, bifurcation diagram, and Poincaré sections are investigated. Then, three control problems: stabilization, synchronization, and coexistence of antisynchronization and complete synchronization are derived with simple yet physically implementable controllers. Finally, numerical simulations are provided to demonstrate effectiveness and the validity of the proposed theoretical results. In conclusion, the main contributions of this paper are given as follows:

(1) A new hyperchaotic finance system is firstly introduced, based on which a new hyperchaos is then generated by setting the parameters.

(2) Dynamic properties of the new generated hyperchaotic finance system are investigated extensively in Section 3, which are important and interesting. It should be pointed out that these dynamic properties results are different from the existing results.

(3) Three control problems of the hyperchaotic finance system are investigated extensively in Section 4. It is noted that the obtained results in this paper have some advantages over the exiting results. In particular, it should be pointed out that the coexistence of antisynchronization and complete synchronization in the two hyperchaotic finance systems is studied, which is a new result.

The rest of this paper is organized as follows. In Section 2, a new hyperchaotic model is introduced, based on which a new hyperchaos is generated by setting the parameters. In Section 3, the dynamic properties of such hyperchaotic finance system are investigated. In Section 4, several control problems of such hyperchaotic finance system are studied extensively, followed by the conclusions in Section 5 .

\section{Model Formulation}

A dynamic model of finance has been reported in [29-31], which is composed of four subblocks, production, money, stock, and labor force, and expressed by four first-order differential equations. The model describes the time variations of four state variables: the interest rate $x$, the investment demand $y$, the price exponent $z$, and the average profit margin $w$. It is well known that the factors affecting the interest rates are related not only to investment demand and price index, but also to the average profit margin. And the average profit margin and interest rate are proportional. Some important results have been obtained. In the next, the four-dimensional hyperchaotic finance system is expressed as follows:

$$
\begin{aligned}
& \dot{x}=z+(y-a) x+w \\
& \dot{y}=1-b y-x^{2} \\
& \dot{z}=-x-c z \\
& \dot{w}=-d x y-k w,
\end{aligned}
$$

where $(x, y, z, w)^{T} \in R^{4}$ is the state vector, $a$ is the saving, $b$ is the per investment cost, $c$ is the elasticity of demands of commercials, and $a, b, c, d, k$ are positive constant parameters.

In order to generate a new hyperchaos, we set $a=0.9, b=$ $0.2, c=1.5, d=0.2$, and select $k$ as a governing parameter according to the two criterions in [7]. The dynamical properties of the hyperchaotic system (1) are firstly studied in Section 3, including symmetry, dissipation, equilibrium point, Lyapunov exponent, Lyapunov dimension, bifurcation diagram, and Poincaré sections. Then, three control problems of such system are investigated in Section 4, that is, stabilization, complete synchronization, and coexistence of antisynchronization and complete synchronization.

\section{Dynamic Properties}

3.1. Symmetry. Since the hyperchaotic system (1) is invariant under the coordinate transformation: $(x, y, z, w) \rightarrow$ $(-x, y,-z,-w)$, the system (1) is symmetric with respect to $z$ axis, which implies that all values of such system parameters are under reflection about $y$-axis. 
3.2. Dissipation. The divergence of this four-dimensional hyperchaotic system (1) is described as

$$
\begin{aligned}
\nabla V & =\frac{\partial \dot{x}}{\partial x}+\frac{\partial \dot{y}}{\partial y}+\frac{\partial \dot{z}}{\partial z}+\frac{\partial \dot{w}}{\partial w}=-(a+b+c+k) \\
& =-(2.8+k)=-u .
\end{aligned}
$$

When $u>0$, that is, $k>-2.8$, thus the system (1) is a dissipative system. It results in that volume element $V_{0} e^{-u t} \rightarrow 0$ as $t \rightarrow \infty$. Therefore, all the trajectories of this four-dimensional hyperchaotic system (1) are ultimately embedded into an chaotic attractor.

3.3. Equilibria. The equilibria of the system (1) are found by solving the following nonlinear equations:

$$
\begin{aligned}
z+(y-a) x+w & =0, \\
1-b y-x^{2} & =0, \\
-x-c z & =0, \\
-d x y-k w & =0 .
\end{aligned}
$$

After computation, it results in

$$
\begin{aligned}
& E_{1}^{*}=\left(0, \frac{1}{b}, 0,0\right), \\
& E_{2}^{*}=\left(\frac{\sqrt{\Delta}_{1}}{\sqrt{\Delta}_{2}},-\frac{\Delta_{3}}{\Delta_{2}},-\frac{\Delta_{1}}{c \sqrt{\Delta}_{2}},-\frac{d(1+a c) \sqrt{\Delta_{1}}}{\Delta_{2}}\right), \\
& E_{3}^{*}=\left(-\frac{\sqrt{\Delta}_{1}}{\sqrt{\Delta}_{2}},-\frac{\Delta_{3}}{\Delta_{2}}, \frac{\Delta_{1}}{c \sqrt{\Delta}_{2}}, \frac{d(1+a c) \sqrt{\Delta_{1}}}{\Delta_{2}}\right),
\end{aligned}
$$

where $\Delta_{1}=-c d-b k+c k-a b c k, \Delta_{2}=-c d+c k, m=-k-a c k$. As an example, set $k=0.17$, the equilibria are given as follows:

$$
\begin{aligned}
& E_{1}^{*}=(0,5,0,0), \\
& E_{2}^{*}=(1.666,-8.87778,-1.11067,17.4004), \\
& E_{3}^{*}=(-1.666,-8.87778,1.11067,-17.4004) .
\end{aligned}
$$

Linearizing the system (1), it results in

$$
J=\left(\begin{array}{cccc}
y-a & x & 1 & 1 \\
2 x & -b & 0 & 0 \\
-1 & 0 & -c & 0 \\
-d y & -d x_{1} & 0 & -k
\end{array}\right) .
$$

For $J_{1}=J\left(E_{1}^{*}\right)$, the eigenvalues can be calculated as $\lambda_{1}=$ 4.1544, $\lambda_{2}=-0.4480, \lambda_{3}=-1.2764, \lambda_{4}=-0.2000$, which implies that $E_{1}^{*}$ is a saddle-focus, thus it is unstable. About $J_{2}=J\left(E_{2}^{*}\right)$ and $J_{3}=J\left(E_{3}^{*}\right)$, the eigenvalues are $\lambda_{1}=-10.0559, \lambda_{2}=-1.5869, \lambda_{3}=-0.0025+0.2871 i$,

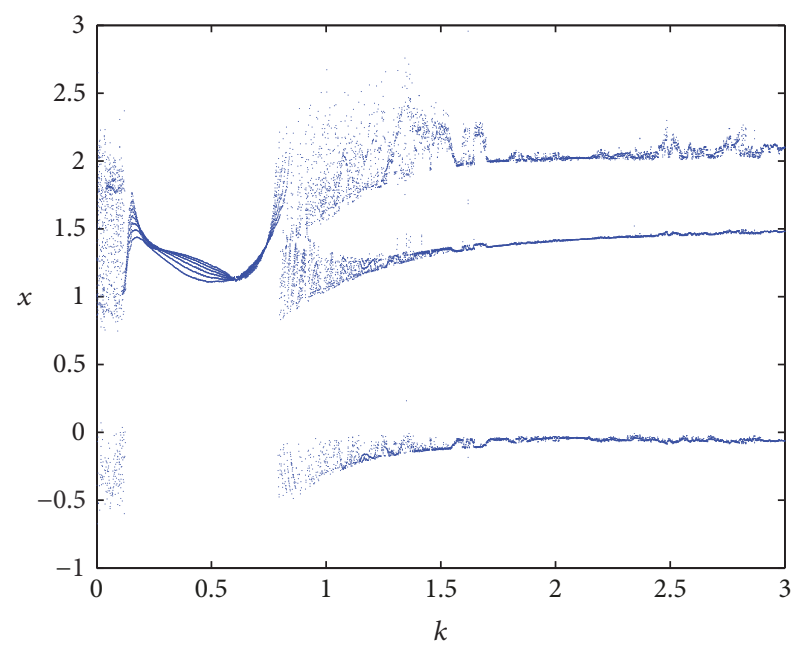

FIgURE 1: Bifurcation diagram of the system (1) with initial conditions $(1,2,0.5,0.5)$ versus parameter $k$.

$\lambda_{4}=-0.0025-0.2871 i, \lambda_{1}=-10.0559, \lambda_{2}=-1.5869, \lambda_{3}=$ $-0.0025+0.2871 i, \lambda_{4}=-0.0025-0.2871 i$, respectively, which imply that $E_{2}^{*}$ and $E_{3}^{*}$ are stable points.

3.4. Bifurcation Diagram. Bifurcation diagram is a useful method to show the dynamical processes of a system with respect to a parameter. In this subsection, the parameter $k$ is chosen as a control parameter. Figure 1 displays the bifurcation diagram of the variable $x$ versus the parameter $k$ which is from 0 to 3 . It is interesting that the evolution procedure of the system (1) through chaotic, hyperchaotic, and periodic orbit, as well as period doubling route to chaos, which displays complex dynamic properties with the increasing of the parament $k$. In order to show the dynamics of the system (1) clearly, we test the time course of the system (1) by selecting several parameters $k$. It can be seen from Figure 2, the state trajectory of the system (1) shows chaos and quasperiodic routes to chaos with the increase of parameter $k$, which is consistent with above results.

3.5. Lyapunov Exponent and Poincaré Sections. For the chaotic systems, Lyapunov exponent is not only an important index to distinguish chaotic attractor, but also a quantitative description of the sensitivity about the initial values. In addition, Poincaré section is also a critical approach to analyze chaotic system; for example, if there exists a closed curve, the movement is quasicycle; if there only exists one fixed point or a few discrete points, the movement is cycle; and if there exists a collection of distribution points along a segment of a line or a curve, the movement is chaos. Here, the Lyapunov exponent and Poincaré sections of the system (1) with $k=3$ are investigated, respectively.

Figure 3 shows the Lyapunov exponent diagram of the hyperchaotic finance system (1). It can be seen that there exist always two positive Lyapunov exponents, which means that the proposed finance system (1) is hyperchaotic. Meanwhile, 

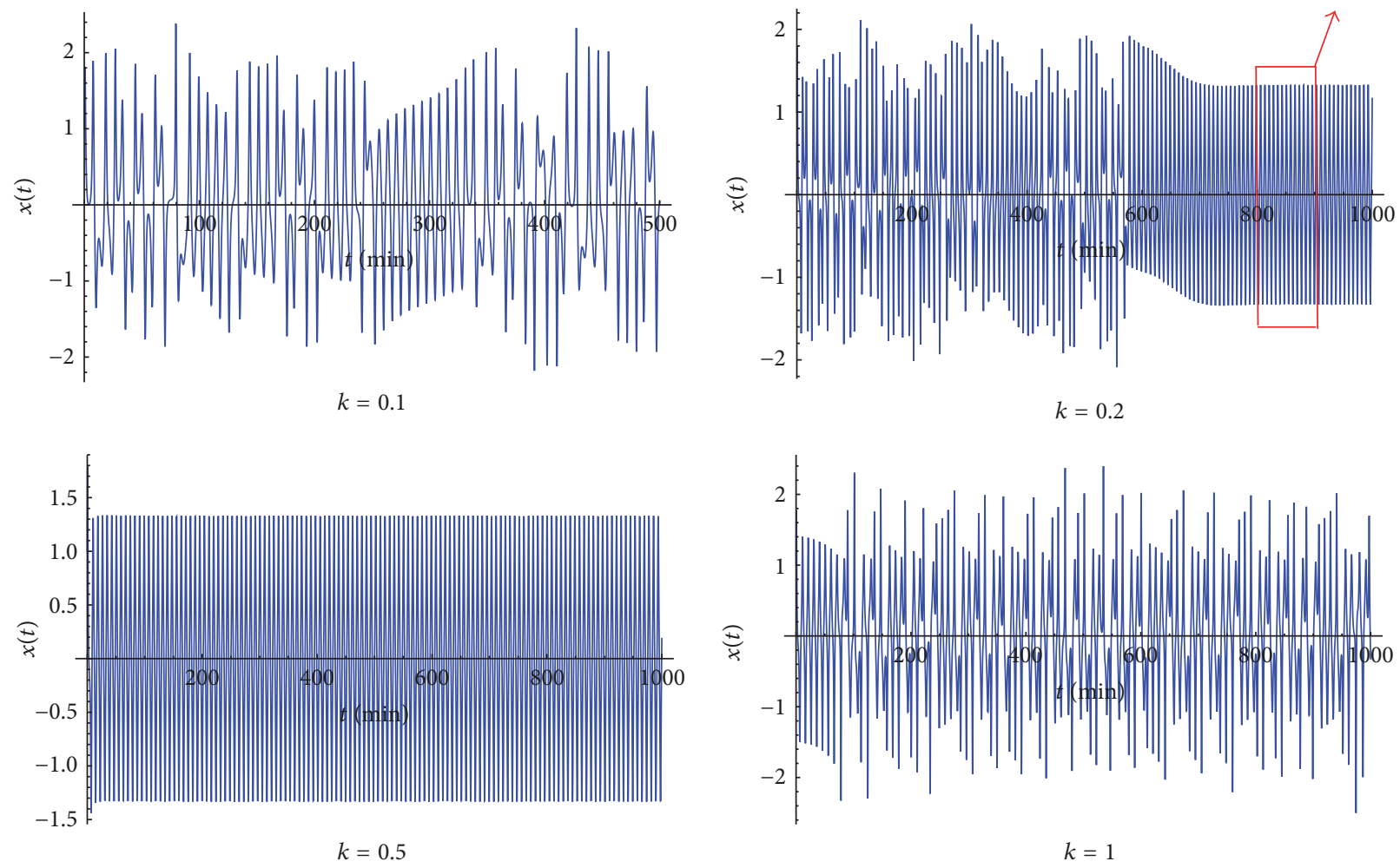

Figure 2: Time course of the system (1) with several fixed $k$ values.

the Poincaré sections in various planes verify the hyperchaotic behavior of the system (1) as shown in Figure 4 . With the following initial conditions: $x(0)=5, y(0)=2, z(0)=$ $-6, w(0)=4$, Figure 5 describes the $3 \mathrm{D}$ projection of the strange attractor of the system (1) on $(x, y, z)$-space, $(x, y, w)$ space, $(y, z, w)$-space, and $(x, w, z)$-space, respectively. In the next, the time course of $x, y, z, w$ is shown with time $t$ with different initial conditions: $x(0)=1, y(0)=2, z(0)=0.5$, $w(0)=0.5$, and $x(0)=1.01, y(0)=2, z(0)=0.5, w(0)=0.5$, respectively. It can be seen, from Figure 6, the motions of the system (1) do not remain similar for long when they are started with very similar initial conditions, which implies that this system is extraordinarily sensitive to initial values.

3.6. Lyapunov Dimension. By Wolf algorithm, the Lyapunov exponents of this four-dimensional hyperchaotic finance system (1) are presented as follows:

$$
\begin{aligned}
& L_{1}=0.034432, \\
& L_{2}=0.018041, \\
& L_{3}=0, \\
& L_{4}=-1.1499 .
\end{aligned}
$$

In addition, the Lyapunov dimension of the system (1) is derived:

$$
\begin{aligned}
D_{L} & =j+\frac{1}{\left|L_{j+1}\right|} \sum_{i=1}^{j} L_{i}=3+\frac{0.034432+0.018041}{1.1499} \\
& =3.045633
\end{aligned}
$$

which is fractional.

\section{Three Control Problems of the New Hyperchaotic Finance System}

In this section, three control problems, stabilization, complete synchronization, and coexistence of antisynchronization and complete synchronization, of the new hyperchaotic finance system (1) are investigated extensively, and some new results are presented.

4.1. Stabilization of the New Hyperchaotic Finance System by a Single Input Controller. For convenience, let $X=\left(x_{1}\right.$, $\left.x_{2}, x_{3}, x_{4}\right)^{T}=(x, y, z, w)^{T}$; then the system (1) is rewritten 

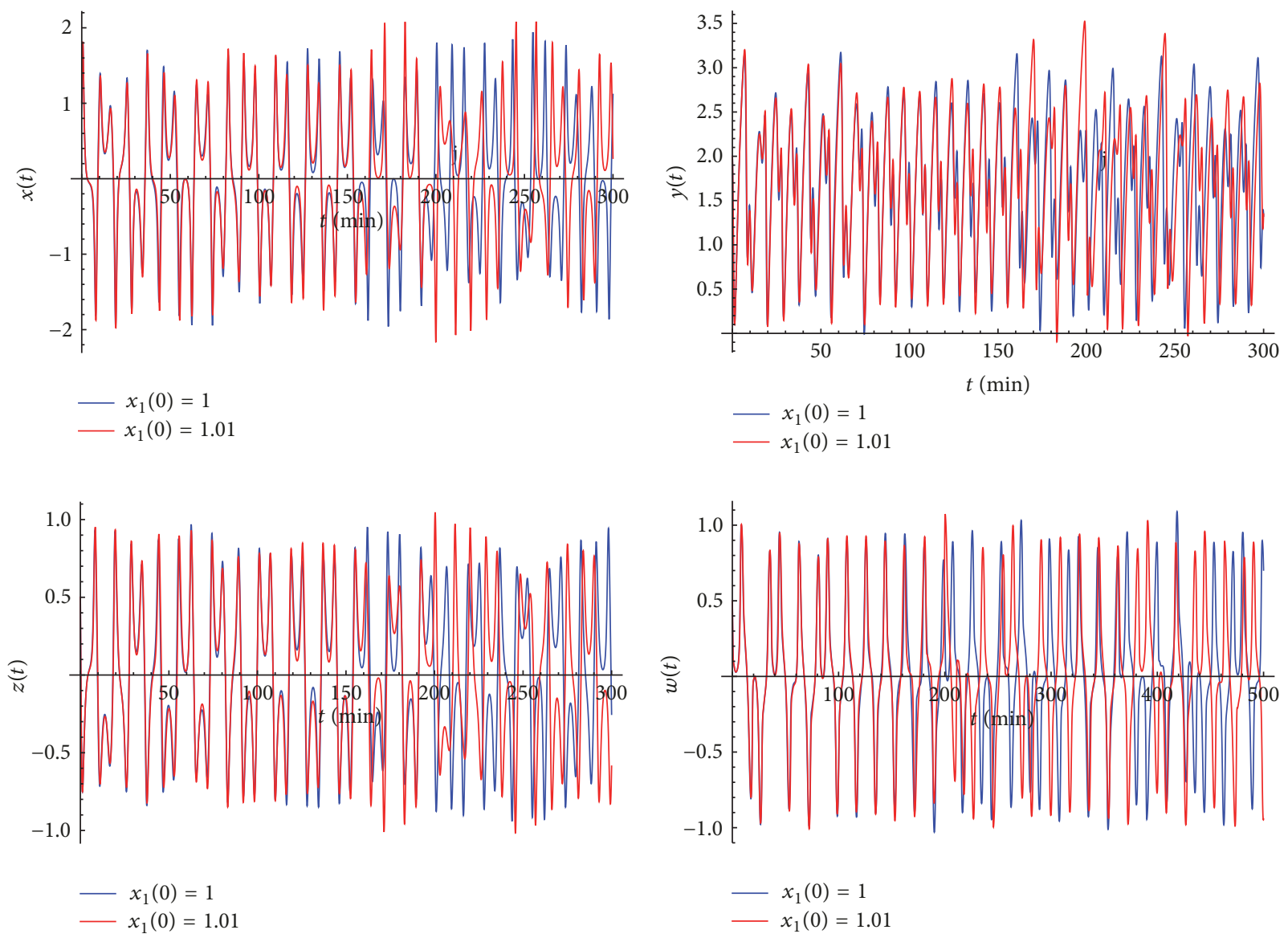

FIGURE 3: Lyapunov exponent of system (1) with varying time $t$.

as

$$
\begin{aligned}
& \dot{x}_{1}=f_{1}(X)=x_{3}+\left(x_{2}-a\right) x_{1}+x_{4} \\
& \dot{x}_{2}=f_{2}(X)=1-b x_{2}-x_{1}^{2} \\
& \dot{x}_{3}=f_{3}(X)=-x_{1}-c x_{3} \\
& \dot{x}_{4}=f_{4}(X)=-d x_{1} x_{2}-k x_{4},
\end{aligned}
$$

where

$$
\begin{aligned}
& a=0.9, \\
& b=0.2, \\
& c=1.5, \\
& d=0.2, \\
& k=0.17 .
\end{aligned}
$$

Remark 1. Since system (9) is hyperchaotic, there exist four positive constants: $\lambda_{i}, i=1,2,3,4$, satisfying

$$
x_{i} f_{i}(X) \leq \lambda_{i} x_{i}^{2}, \quad i=1,2,3,4 .
$$

According to Section 3.3, $E_{1}^{*}=(0,5,0,0)$ is an equilibrium point of the hyperchaotic finance system (9). Next, the stabilization problem of system (9) will be studied, that is, designing a physically implementable controller $u$ to force the states of such system to the equilibrium point $E_{1}^{*}$.

Consider system (9). Obviously, if $x_{1}=0$, then the following subsystem

$$
\begin{aligned}
& \dot{x}_{2}=1-b x_{2} \\
& \dot{x}_{3}=-c x_{3} \\
& \dot{x}_{4}=-k x_{4}
\end{aligned}
$$

is globally asymptotically stable (GAS) with respect to $E_{1}^{*}$.

Thus, the controller is designed as

$$
u=\left(\begin{array}{l}
u_{1} \\
u_{2} \\
u_{3} \\
u_{4}
\end{array}\right)=\left(\begin{array}{c}
k_{1} x_{1} \\
0 \\
0 \\
0
\end{array}\right)
$$



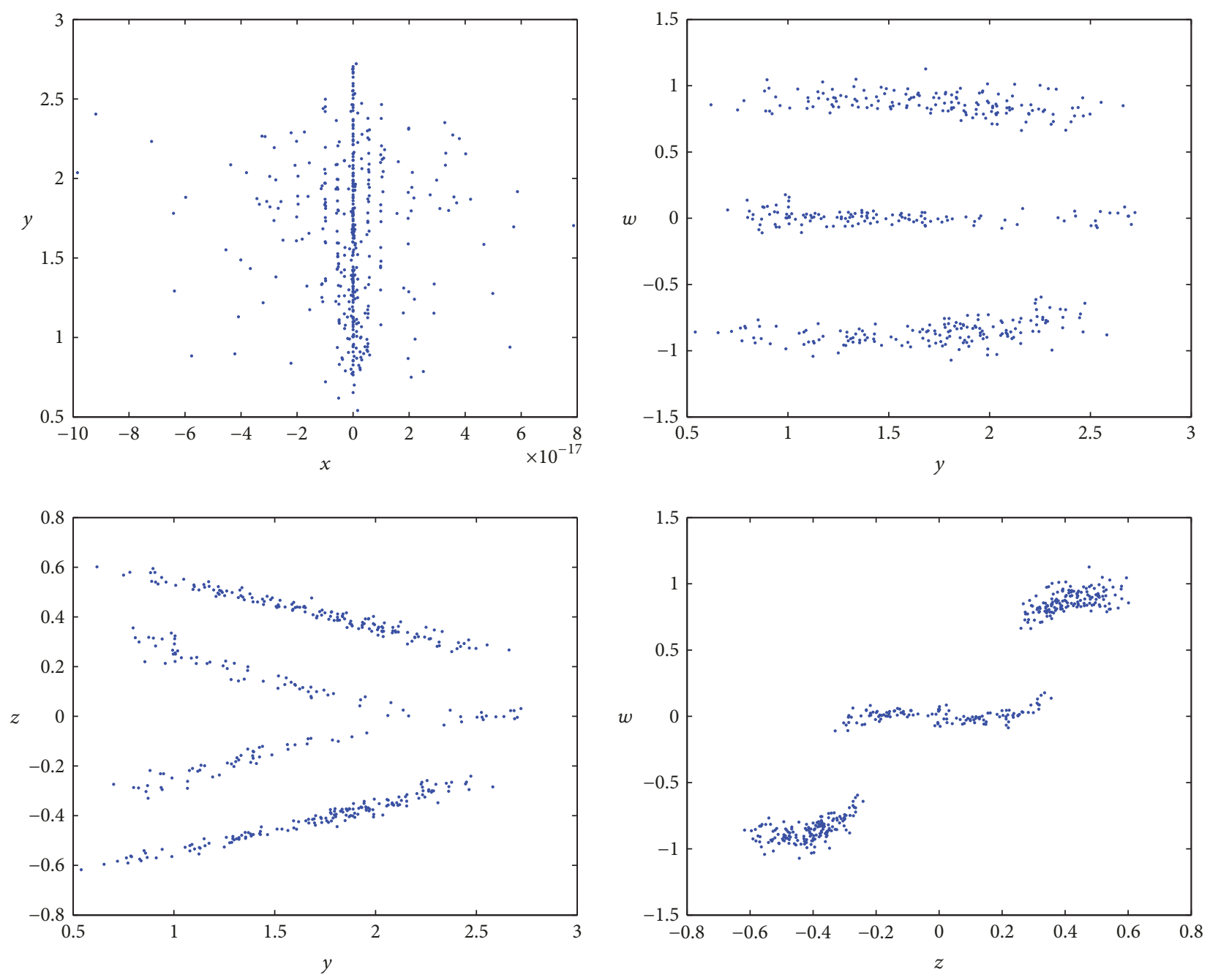

FIGURE 4: Poincaré sections of system (1).

and controlled system (9) is presented as

$$
\begin{aligned}
& \dot{x}_{1}=f_{1}(X)+u_{1}=x_{3}+\left(x_{2}-a\right) x_{1}+x_{4}+k_{1} x_{1} \\
& \dot{x}_{2}=f_{2}(X)+u_{2}=1-b x_{2}-x_{1}^{2} \\
& \dot{x}_{3}=f_{3}(X)+u_{3}=-x_{1}-c x_{3} \\
& \dot{x}_{4}=f_{4}(X)+u_{4}=-d x_{1} x_{2}-k x_{4},
\end{aligned}
$$

where the dynamic gain $k_{1}$ is evolved by the following law:

$$
\dot{k}_{1}=-\gamma x_{1}^{2} \text {, }
$$

where $\gamma>0$ which is chosen in advance.

Remark 2. In [30], the obtained controller is given as

$$
u=\left(\begin{array}{l}
u_{1} \\
u_{2} \\
u_{3} \\
u_{4}
\end{array}\right)=\left(\begin{array}{c}
-k_{2} x_{1} \\
-k_{2} y_{1} \\
0 \\
-k_{2} z_{1}
\end{array}\right) .
$$

Obviously, controller (16) is more complicated than controller (13).

For convenience, systems (14) and (15) are called the auxiliary system, and a candidate Lyapunov function is introduced as follows:

$$
V\left(x, k_{1}\right)=\frac{1}{2} X^{T} X+\frac{1}{2 \gamma}\left(k_{1}+L_{1}\right)^{2},
$$

where

$$
L_{1}>M_{1} \sup _{x_{1} \neq 0} \frac{X^{T} X}{x_{1}^{2}}, \quad M_{1}=\max _{i=1}^{4} \lambda_{i}
$$

Then, the following conclusion is presented.

Theorem 3. For any initial values, the orbits $X(t)$ of the system (14) converge to the equilibrium point $E_{1}^{*}$ as $t \rightarrow \infty$; that is, the stabilization of system (9) is achieved by the above controller (13). 

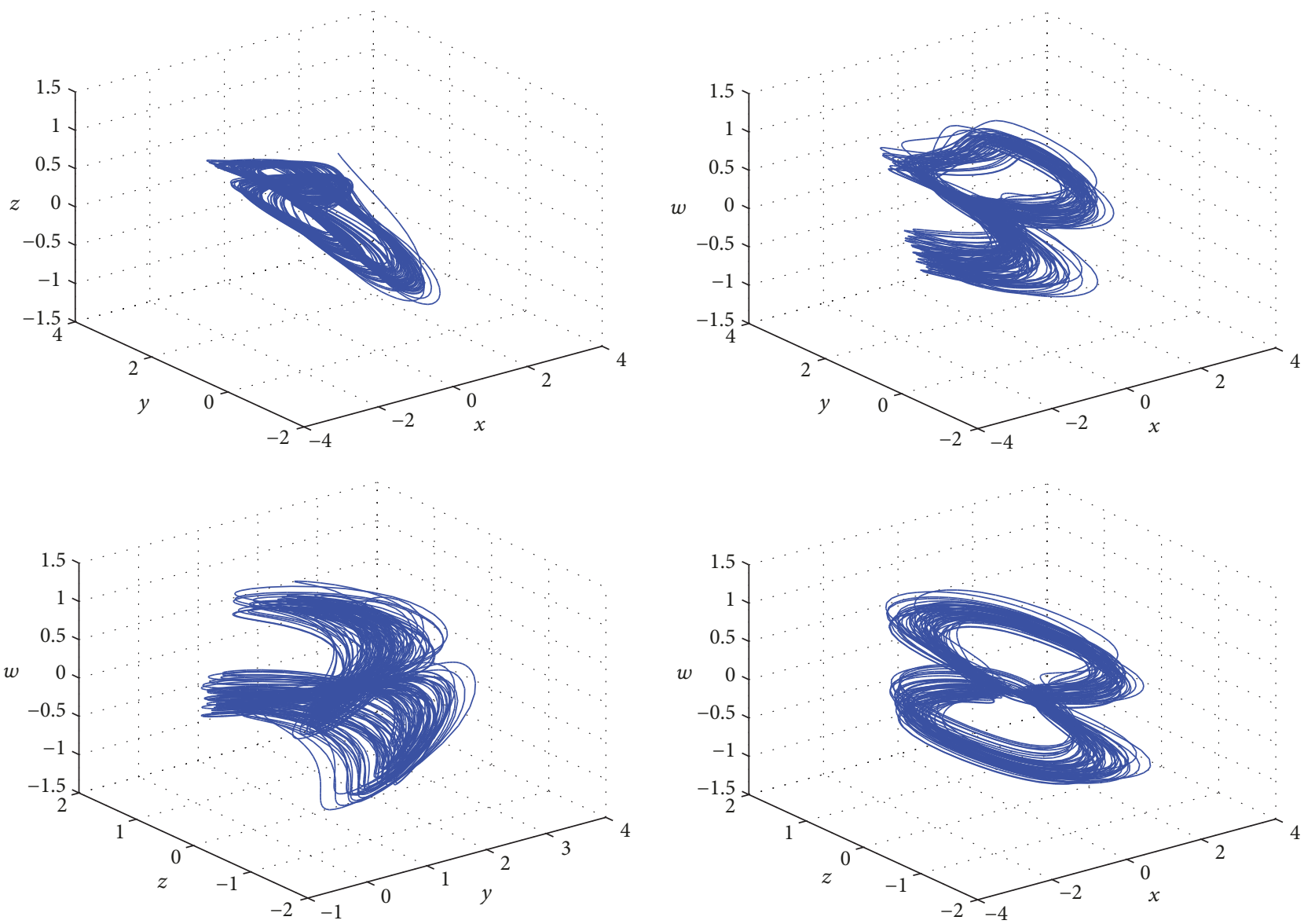

FIGURE 5: 3D projection of the strange attractor of system (1) on $(x, y, z)$-space, $(x, y, w)$-space, $(y, z, w)$-space, and $(x, w, z)$-space, respectively.

Proof. The time derivative of $V$ with respect to the auxiliary system is given as follows:

$$
\begin{aligned}
\dot{V} & =X^{T} \dot{X}+\frac{1}{\gamma}\left(k_{1}+L_{1}\right) \dot{k}_{1} \\
& =x_{1}\left(f_{1}(X)+k_{1} x_{1}\right)+\sum_{i=2}^{4} x_{i} f_{i}(X)-\left(k_{1}+L_{1}\right) x_{1}^{2} \\
& =\sum_{i=1}^{4} x_{i} f_{i}(X)-L_{1} x_{1}^{2} \leq M_{1} X^{T} X-L_{1} x_{1}^{2}<0 .
\end{aligned}
$$

Thus the conclusion is proved, which completes the proof.

Numerical simulation is carried out with the following initial conditions: $x_{1}(0)=1, x_{2}(0)=2, x_{3}(0)=-4, x_{4}(0)=$ -2 and $k_{1}(0)=-1, \gamma=1$. Figure 7 displays that system (9) is stabilized to its equilibrium point $E_{1}^{*}$.

4.2. Complete Synchronization of the Two Hyperchaotic Finance Systems by a Single Input Controller. In this subsection, the complete synchronization of two hyperchaotic finance systems with different initial conditions are investigated, and some new results are presented in the following.
Make system (9) the master system; then the corresponding uncontrolled (i.e., $u=0$ ) slave system with variable $Y$ being given as

$$
\begin{aligned}
& \dot{y}_{1}=f_{1}(Y)=y_{3}+\left(y_{2}-a\right) y_{1}+y_{4} \\
& \dot{y}_{2}=f_{2}(Y)=1-b y_{2}-y_{1}^{2} \\
& \dot{y}_{3}=f_{3}(Y)=-y_{1}-c y_{3} \\
& \dot{y}_{4}=f_{4}(Y)=-d y_{1} y_{2}-k y_{4},
\end{aligned}
$$

where $Y=\left(y_{1}, y_{2}, y_{3}, y_{4}\right)^{T}$ is the state vector and $a, b, c, d, k$ are given as (10).

Let $e=\left(e_{1}, e_{2}, e_{3}, e_{4}\right)^{T}=Y-X$; then the uncontrolled (i.e., $u=0$ ) error system is described as

$$
\begin{aligned}
& \dot{e}_{1}=F_{1}(X, e)=-a e_{1}+x_{1} e_{2}+x_{2} e_{1}+e_{1} e_{2}+e_{3}+e_{4} \\
& \dot{e}_{2}=F_{2}(X, e)=-b e_{2}-2 x_{1} e_{1}-e_{1}^{2} \\
& \dot{e}_{3}=F_{3}(X, e)=-e_{1}-c e_{3} \\
& \dot{e}_{4}=F_{4}(X, e)=-d\left(e_{1} x_{2}+e_{2} x_{1}+e_{1} e_{2}\right)-k e_{4} .
\end{aligned}
$$



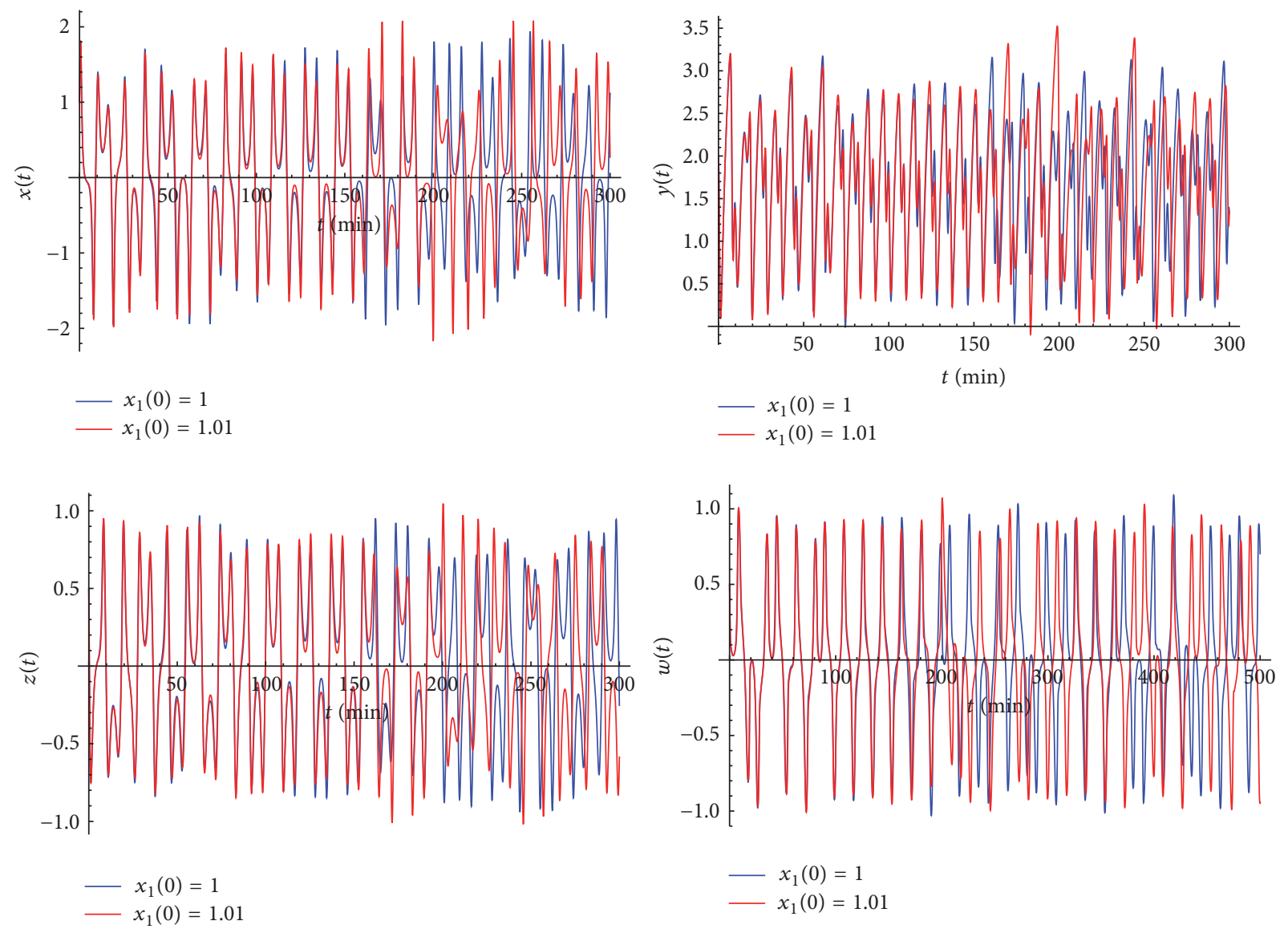

Figure 6: Time course of $x, y, z, w$ of system (1) with similar initial values.

Remark 4. Since system (9) is hyperchaotic, there exist four positive constants: $l_{i}, i=1,2,3,4$, such that

$$
e_{i} F_{i}(X, e) \leq l_{i} e_{i}^{2}, \quad i=1,2,3,4
$$

Consider system (21). Obviously, if $e_{1}=0$, then the following subsystem

$$
\begin{aligned}
& \dot{e}_{2}=-b e_{2} \\
& \dot{e}_{3}=-c e_{3} \\
& \dot{e}_{4}=-d e_{2} x_{1}-k e_{4}
\end{aligned}
$$

is GAS with respect to origin.

Thus, the designed controller is given as

$$
u=\left(\begin{array}{l}
u_{1} \\
u_{2} \\
u_{3} \\
u_{4}
\end{array}\right)=\left(\begin{array}{c}
k_{2} e_{1} \\
0 \\
0 \\
0
\end{array}\right),
$$

and the controlled error system is described as

$$
\begin{aligned}
\dot{e}_{1} & =F_{1}(X, e)+u_{1} \\
& =-a e_{1}+x_{1} e_{2}+x_{2} e_{1}+e_{1} e_{2}+e_{3}+e_{4}+k_{2} e_{2} \\
\dot{e}_{2} & =F_{2}(X, e)+u_{2}=-b e_{2}-2 x_{1} e_{1}-e_{1}^{2} \\
\dot{e}_{3} & =F_{3}(X, e)+u_{3}=-e_{1}-c e_{3} \\
\dot{e}_{4} & =F_{4}(X, e)+u_{4}=-d\left(e_{1} x_{2}+e_{2} x_{1}+e_{1} e_{2}\right)-k e_{4},
\end{aligned}
$$

where the dynamic gain $k_{2}$ is evolved by the following law:

$$
\dot{k}_{2}=-\gamma e_{1}^{2} \text {, }
$$

where $\gamma>0$ which is chosen in advance.

Similarly, systems (25) and (26) are called the auxiliary system, and a candidate Lyapunov function is presented as follows:

$$
V\left(e, k_{2}\right)=\frac{1}{2} e^{T} e+\frac{1}{2 \gamma}\left(k_{2}+L_{2}\right)^{2}
$$

where

$$
L_{2}>M_{2} \sup _{e_{1} \neq 0} \frac{e^{T} e}{e_{1}^{2}}, \quad M_{2}=\max _{i=1}^{4} l_{i} .
$$

Next, a conclusion is obtained. 

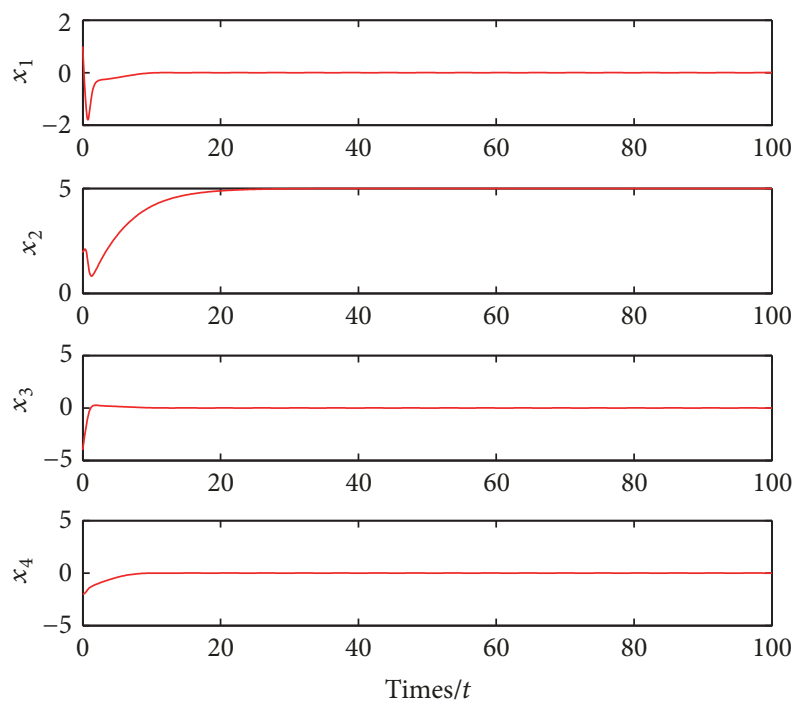

Figure 7: The figure shows that the orbit $X(t)$ of the system (14) converges to $(0,5,0,0)^{T}$ as $t \rightarrow \infty$.

Theorem 5. For any initial values, the orbits $e(t)$ of the error system (25) converge to origin as $\rightarrow \rightarrow \infty$, that is, master system (9) synchronizes slave system (20) by the above controller (24).

Proof. The time derivative of $V$ with respect to the auxiliary system is given as follows:

$$
\begin{aligned}
\dot{V}= & e^{T} \dot{e}+\frac{1}{\gamma}\left(k_{2}+L_{2}\right) \dot{k}_{2} \\
= & e_{1}\left(F_{1}(X, e)+k_{2} e_{1}\right)+\sum_{i=2}^{4} e_{i} F_{i}(X, e) \\
& -\left(k_{2}+L_{2}\right) e_{1}^{2}=\sum_{i=1}^{4} e_{i} F_{i}(X, e)-L_{2} e_{1}^{2} \\
\leq & M_{2} e^{T} e-L_{2} e_{1}^{2}<0 .
\end{aligned}
$$

Thus the result is obtained, which completes the proof.

Similarly, numerical simulation is carried out with the following initial conditions: $x_{1}(0)=1, x_{2}(0)=2, x_{3}(0)=$ $-4, x_{4}(0)=-1, y_{1}(0)=-2, y_{2}(0)=-1, y_{3}(0)=$ $1, y_{4}(0)=1$, and $k_{1}(0)=-1, \gamma=1$. Figure 8 shows that the complete synchronization of two hyperchaotic finance systems with different initial conditions being achieved by the above controller (24).

4.3. Coexistence of Antisynchronization and Complete Synchronization in the Two Hyperchaotic Finance Systems. In this section, the coexistence of antisynchronization and complete synchronization in the two hyperchaotic finance systems is investigated, and some new results are obtained in the following.
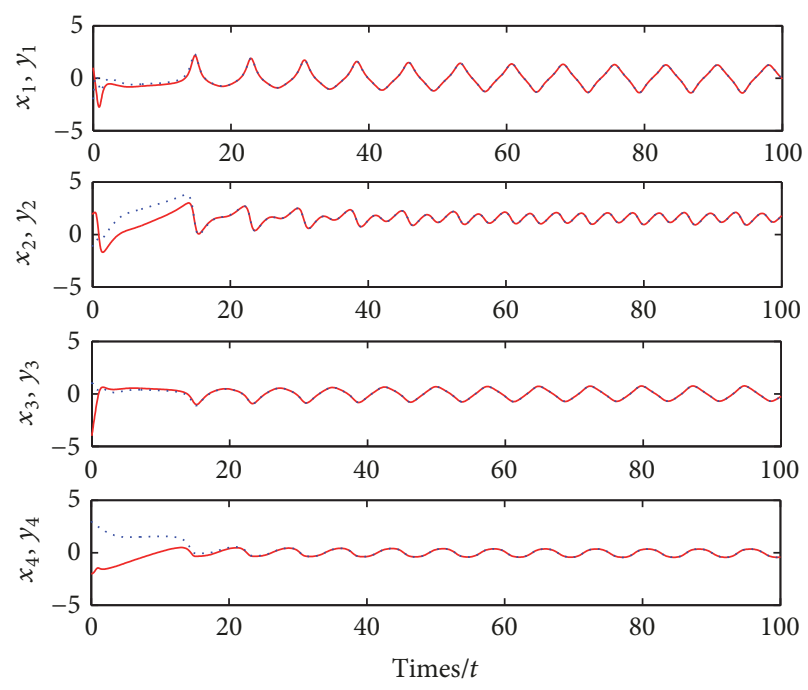

FIgURE 8: The figure shows that the states, $x_{1}, x_{2}, x_{3}, x_{4}$, synchronize the states, $y_{1}, y_{2}, y_{3}, y_{4}$, respectively.

According to the results in [26], we should set

$$
\begin{aligned}
& E_{1}=y_{1}+x_{1}, \\
& e_{2}=y_{2}-x_{2}, \\
& E_{3}=y_{3}+x_{3}, \\
& E_{4}=y_{4}+x_{4} .
\end{aligned}
$$

Then, the uncontrolled (i.e., $u=0$ ) combination (including the sum $E$ and the error $e_{2}$ ) system is described as follows:

$$
\begin{aligned}
\dot{E}_{1} & =G_{1}\left(X, E, e_{2}\right) \\
& =-a E_{1}+E_{3}+E_{1} e_{2}+x_{2} E_{1}-x_{1} e_{2}+E_{4} \\
\dot{e}_{2} & =G_{2}\left(X, E, e_{2}\right)=-b e_{2}-E_{1}^{2}+2 x_{1} E_{1} \\
\dot{E}_{3} & =G_{3}\left(X, E, e_{2}\right)=-E_{1}-c E_{3} \\
\dot{E}_{3} & =G_{4}\left(X, E, e_{2}\right)=-d\left(E_{1} e_{2}+x_{2} E_{1}-x_{1} e_{2}\right)-k E_{4},
\end{aligned}
$$

where $E=\left(E_{1}, E_{3}, E_{4}\right)^{T}$.

Remark 6. Since system (9) is hyperchaotic, there exist four positive constants: $n_{i}, i=1,2,3,4$ satisfying

$$
\begin{aligned}
E_{i} G_{i}\left(X, E, e_{2}\right) \leq n_{i} E_{i}^{2}, & \\
i & =1,3,4, e_{2} G_{2}\left(X, E, e_{2}\right) \leq n_{2} e_{2}^{2} .
\end{aligned}
$$

Consider the combination system (31). Obviously, if $E_{1}=$ 0 , then the following subsystem

$$
\begin{aligned}
& \dot{e}_{2}=-b e_{2} \\
& \dot{E}_{3}=-E_{1}-c E_{3} \\
& \dot{E}_{4}=-2 x_{1} d e_{2}-k E_{4}
\end{aligned}
$$

is GAS with respect to origin. 
Therefore, the designed controller is expressed as

$$
u=\left(\begin{array}{l}
u_{1} \\
u_{2} \\
u_{3} \\
u_{4}
\end{array}\right)=\left(\begin{array}{c}
k_{3} E_{1} \\
0 \\
0 \\
0
\end{array}\right),
$$

and the controlled combination system is presented as follows:

$$
\begin{aligned}
\dot{E}_{1} & =G_{1}\left(X, E, e_{2}\right) \\
& =-a E_{1}+E_{3}+E_{1} e_{2}+x_{2} E_{1}-x_{1} e_{2}+E_{4}+k_{3} E_{1} \\
\dot{e}_{2} & =G_{2}\left(X, E, e_{2}\right)=-b e_{2}-E_{1}^{2}+2 x_{1} E_{1} \\
\dot{E}_{3} & =G_{3}\left(X, E, e_{2}\right)=-E_{1}-c E_{3} \\
\dot{E}_{3} & =G_{4}\left(X, E, e_{2}\right)=-d\left(E_{1} e_{2}+x_{2} E_{1}-x_{1} e_{2}\right)-k E_{4},
\end{aligned}
$$

where the dynamic gain $k_{3}$ is evolved by the following law:

$$
\dot{k}_{3}=-\gamma E_{1}^{2} \text {, }
$$

where $\gamma>0$ which is chosen in advance.

Similarly, systems (35) and (36) are called the auxiliary system, and a candidate Lyapunov function is introduced as follows:

$$
V\left(E, e_{2}, k_{3}\right)=\frac{1}{2}\left(e_{2}^{2}+E^{T} E\right)+\frac{1}{2 \gamma}\left(k_{3}+L_{3}\right)^{2},
$$

where

$$
L_{3}>M_{3} \sup _{E_{1} \neq 0} \frac{e_{2}^{2}+E^{T} E}{E_{1}^{2}}, \quad M_{3}=\max _{i=1}^{4} n_{i}
$$

Next, a conclusion is presented as follows.

Theorem 7. For any initial values, the orbits $\left(E(t), e_{2}(t)\right)^{T}$ of the combination system (35) converge to origin as $t \rightarrow \infty$, which implies that the coexistence of antisynchronization and complete synchronization in the two new hyperchaotic finance systems is achieved by the above controller (34), that is, states $x_{1}, x_{3}, x_{4}$ antisynchronize states $y_{1}, y_{3}, y_{4}$, while rest state $x_{2}$ synchronizes state $y_{2}$, respectively.

Proof. The time derivative of $V$ with respect to the auxiliary system is given as follows:

$$
\begin{aligned}
\dot{V} & =E^{T} \dot{E}+e_{2} \dot{e}_{2}+\frac{1}{\gamma}\left(k_{3}+L_{3}\right) \dot{k}_{3} \\
& =\sum_{i=1, \neq 2}^{4} E_{i} G_{i}\left(X, E, e_{2}\right)+e_{2} G_{2}\left(X, E, e_{2}\right)-L_{3} E_{1}^{2} \\
& \leq M_{3}\left(E^{T} E+e_{2}^{2}\right)-L_{3} E_{1}^{2}<0 .
\end{aligned}
$$

Thus the conclusion holds, which completes the proof.

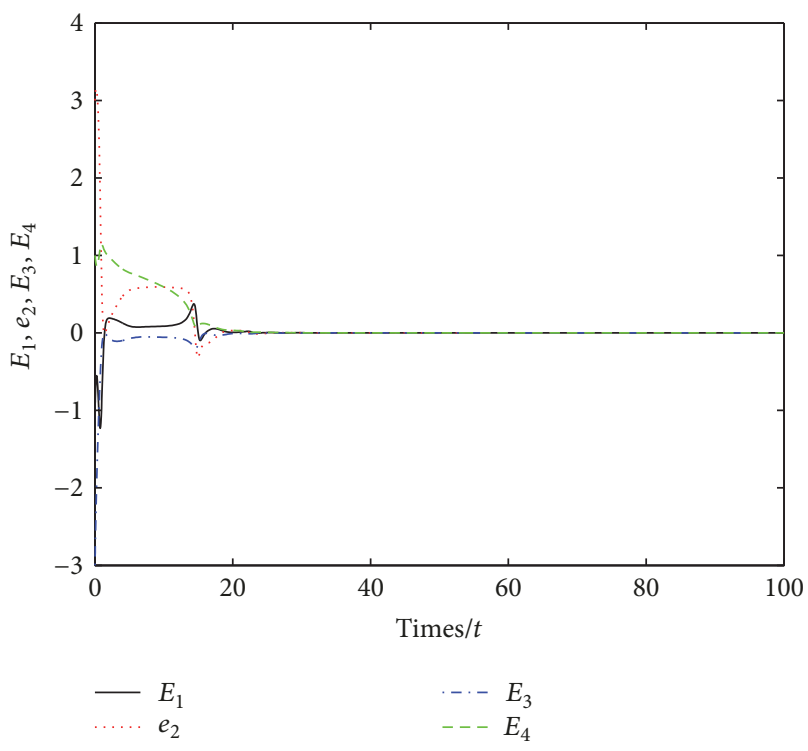

Figure 9: The figure shows that the orbits $\left(E(t), e_{2}(t)\right)^{T}$ of the combination system (35) converge to origin as $t \rightarrow \infty$.
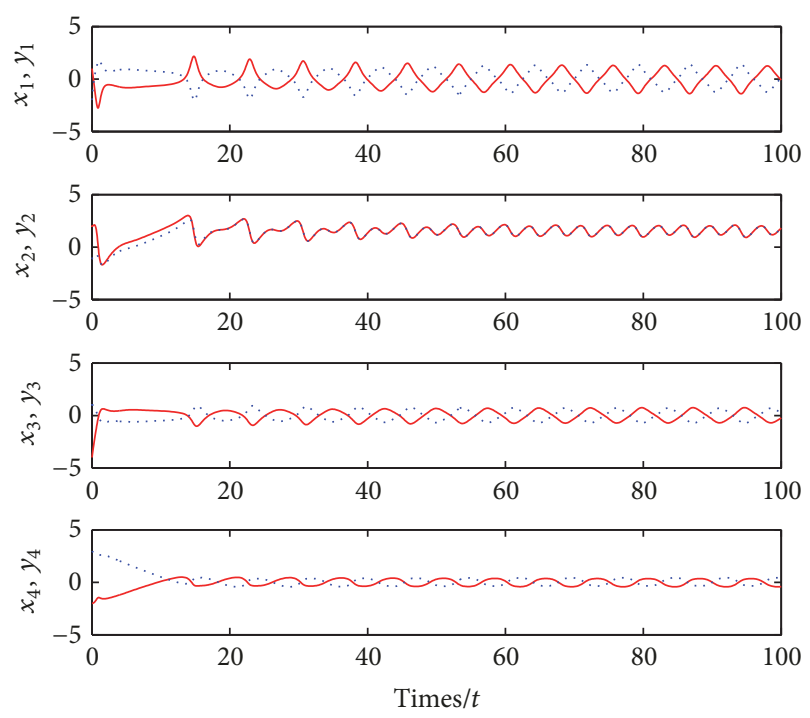

Figure 10: The figure shows that states $x_{1}, x_{3}, x_{4}$ antisynchronize states $y_{1}, y_{3}, y_{4}$, while state $x_{2}$ synchronizes state $y_{2}$, respectively.

Similarly, numerical simulation is carried out with the following initial conditions: $x_{1}(0)=1, x_{2}(0)=2, x_{3}(0)=$ $-4, x_{4}(0)=-1, y_{1}(0)=-2, y_{2}(0)=-1, y_{3}(0)=1, y_{4}(0)=$ 1 , and $k_{1}(0)=-1, \gamma=1$. Figure 9 displays that the orbits $\left(E(t), e_{2}(t)\right)^{T}$ of the combination system (35) converge to origin as $t \rightarrow \infty$, which implies that the coexistence of antisynchronization and complete synchronization in the two new hyperchaotic finance systems is achieved by the above controller (34). Figure 10 shows that the states $x_{1}, x_{3}, x_{4}$ antisynchronize the states $y_{1}, y_{3}, y_{4}$, while state $x_{2}$ synchronizes state $y_{2}$, respectively. 


\section{Conclusions}

In this paper, a new four-dimensional hyperchaotic finance system has been introduced, based on which a new hyperchaos has been generated. This system has simple algebraic structure and can display complex characteristics: chaotic, hyperchaotic, and quasiperiodic phenomena occur alternately versus parameters. Then, the dynamic properties including symmetry, dissipation, equilibrium point, Lyapunov exponent, bifurcation diagram, and Poincaré sections are studied. Furthermore, three control problems of such system have been achieved by three single input controllers, respectively. Compared to the existing ones, the designed controllers in this paper are simpler. Finally, the validity and the effectiveness of the obtained theoretical results have been verified by the provided numerical simulations.

\section{Conflicts of Interest}

The author declares that there are no financial and personal relationships with other people or organizations that can inappropriately influence this work; there is no professional or other personal interest of any nature or kind in any product, service, and/or company that could be construed as influencing the position presented in, or the review of, this article. The author declares that there are no conflicts of interest regarding the publication of this paper.

\section{Acknowledgments}

The author would like acknowledge the School of Business Administration at Andrews University for providing wonderful research environment. In particular, the help of Dr. Ralph Trecartin, Dr. Jerry Chi, and the colleagues during the current research is highly appreciated. This paper is supported by the Social Science Foundation of Shandong Province (17CLYJ43).

\section{References}

[1] E. N. Lorenz, "Deterministic nonperiodic flow," Journal of the Atmospheric Sciences, vol. 20, no. 2, pp. 130-141, 1963.

[2] O. E. Rössler, "An equation for continuous chaos," Physics Letters A, vol. 57, no. 5, pp. 397-398, 1976.

[3] J. H. Ma and Y. S. Chen, "study for the bifurcation topological structure and the global complicated character of a kind of nonlinear finance system (I)," Applied Mathematics and Mechanics, vol. 22, no. 11, pp. 1240-1251, 2001.

[4] H.-P. Ren, M. S. Baptista, and C. Grebogi, "Wireless communication with chaos," Physical Review Letters, vol. 110, no. 18, Article ID 184101, 2013.

[5] G. Bhatnagar and Q. M. Jonathan Wu, "A novel chaos-based secure transmission of biometric data," Neurocomputing, vol. 147, no. 1, pp. 444-455, 2015.

[6] L. Liu and R. Guo, "Control problems of Chen-Lee system by adaptive control method," Nonlinear Dynamics, vol. 87, no. 1, pp. 503-510, 2017.

[7] O. E. Rossler, "An equation for hyperchaos," Physics Letters A, vol. 71, no. 2-3, pp. 155-157, 1979.
[8] S. Mukherjee, S. K. Palit, S. Banerjee, A. W. A. Wahab, M. R. K. Ariffin, and D. K. Bhattacharya, "Computing two dimensional Poincaré maps for hyperchaotic dynamics," Applied Mathematics and Computation, vol. 301, pp. 140-154, 2017.

[9] A. Mohammadzadeh and S. Ghaemi, "Synchronization of uncertain fractional-order hyperchaotic systems by using a new self-evolving non-singleton type-2 fuzzy neural network and its application to secure communication," Nonlinear Dynamics, vol. 88 , no. $1,2017$.

[10] E. Ott, C. Grebogi, and J. A. Yorke, "Controlling chaos," Physical Review Letters, vol. 64, no. 11, pp. 1196-1199, 1990.

[11] L. M. Pecora and T. L. Carroll, "Synchronization in chaotic systems," Physical Review Letters, vol. 64, no. 8, pp. 821-824, 1990.

[12] D. Auerbach, C. Grebogi, E. Ott, and J. A. Yorke, "Controlling chaos in high-dimensional systems," Physical Review Letters, vol. 69, no. 24, pp. 3479-3482, 1992.

[13] J. Sieber, O. E. Omel'Chenko, and M. Wolfrum, "Controlling unstable chaos: stabilizing chimera states by feedback," Physical Review Letters, vol. 112, Article ID 054102, 2014.

[14] D. Huang, "Simple adaptive-feedback controller for identical chaos synchronization," Physical Review E: Statistical, Nonlinear, and Soft Matter Physics, vol. 71, Article ID 037203, 2005.

[15] R. Guo, "A simple adaptive controller for chaos and hyperchaos synchronization," Physics Letters A, vol. 372, no. 34, pp. 55935597, 2008.

[16] M. G. Rosenblum, A. S. Pikovsky, and J. Kurths, "Phase synchronization of chaotic oscillators," Physical Review Letters, vol. 76, no. 11, pp. 1804-1807, 1996.

[17] A. S. Pikovsky, M. G. Rosenblum, G. V. Osipov, and J. Kurths, "Phase synchronization of chaotic oscillators by external driving," Physica D: Nonlinear Phenomena, vol. 104, no. 3-4, pp. 219238, 1997.

[18] M. G. Rosenblum, A. S. Pikovsky, and J. Kurths, "From phase to lag synchronization in coupled chaotic oscillators," Physical Review Letters, vol. 78, no. 22, pp. 4193-4196, 1997.

[19] S. Taherion and Y.-C. Lai, "Observability of lag synchronization of coupled chaotic oscillators," Physical Review E: Statistical Physics, Plasmas, Fluids, and Related Interdisciplinary Topics, vol. 59, no. 6, pp. R6247-R6250, 1999.

[20] N. F. Rulkov, M. M. Sushchik, L. S. Tsimring, and H. D. I. Abarbanel, "Generalized synchronization of chaos in directionally coupled chaotic systems," Physical Review E: Statistical, Nonlinear, and Soft Matter Physics, vol. 51, no. 2, pp. 980-994, 1995.

[21] H. D. I. Abarbanel, N. F. Rulkov, and M. M. Sushchik, "Generalized synchronization of chaos: the auxiliary system approach," Physical Review E, vol. 53, no. 5, article 4528, 1996.

[22] S. Hammami, M. Benrejeb, M. Feki, and P. Borne, "Feedback control design for Rössler and Chen chaotic systems antisynchronization," Physics Letters A, vol. 374, no. 28, pp. 28352840, 2010.

[23] L. Ren and R. W. Guo, "A necessary and sufficient condition of anti-synchronization for chaotic systems and its applications," Mathematical Problems in Engineering, vol. 2015, Article ID 434651, pp. 1-7, 2015.

[24] R. Mainieri and J. Rehacek, "Projective synchronization in three-dimensional chaotic systems," Physical Review Letters, vol. 82, no. 15, pp. 3042-3045, 1999.

[25] D. Xu, "Control of projective synchronization in chaotic systems," Physical Review E: Statistical, Nonlinear, and Soft Matter Physics, vol. 63, Article ID 027201, pp. 1-4, 2001. 
[26] L. Ren, R. Guo, and U. E. Vincent, "Coexistence of synchronization and anti-synchronization in chaotic systems," Archives of Control Sciences, vol. 26, no. 1, pp. 69-79, 2016.

[27] R.-W. Guo, "Simultaneous synchronization and antisynchronization of two identical new 4D chaotic systems," Chinese Physics Letters, vol. 28, no. 4, Article ID 040205, 2011.

[28] J.-M. Grandmont, "On endogenous competitive business cycles," Econometrica, vol. 53, no. 5, pp. 995-1045, 1985.

[29] H. Yu, G. Cai, and Y. Li, "Dynamic analysis and control of a new hyperchaotic finance system," Nonlinear Dynamics, vol. 67, no. 3, pp. 2171-2182, 2012.

[30] G. Cai, P. Hu, and Y. Li, "Modified function lag projective synchronization of a financial hyperchaotic system," Nonlinear Dynamics, vol. 69, no. 3, pp. 1457-1464, 2012.

[31] W. Wu and Z. Chen, "Hopf bifurcation and intermittent transition to hyperchaos in a novel strong four-dimensional hyperchaotic system," Nonlinear Dynamics, vol. 60, no. 4, pp. 615-630, 2010. 


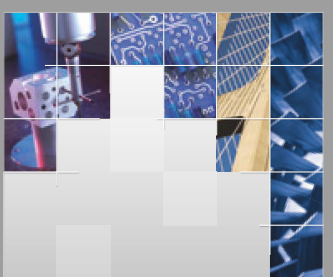

\section{Enfincering}
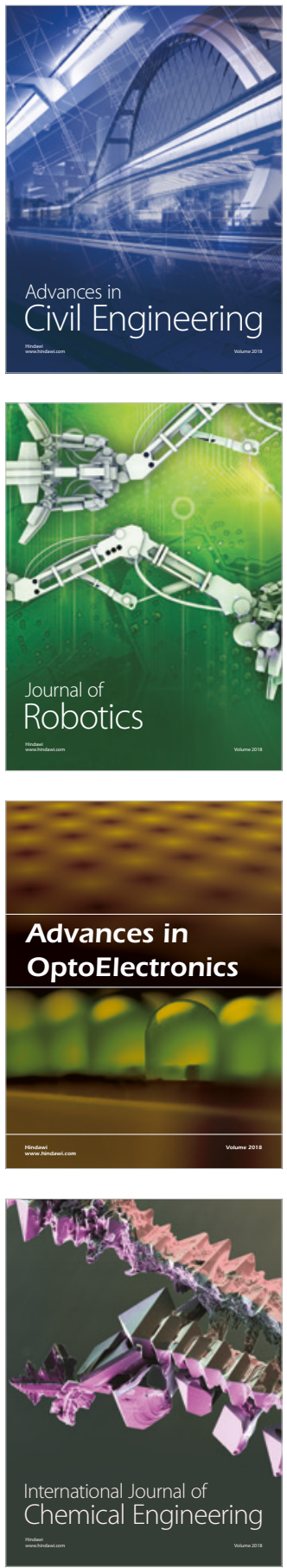

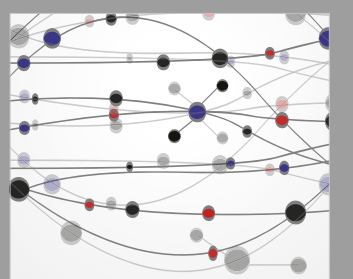

\section{Rotating \\ Machinery}

The Scientific World Journal

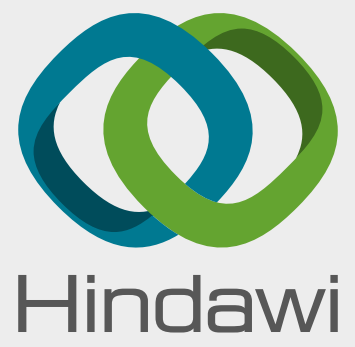

Submit your manuscripts at

www.hindawi.com
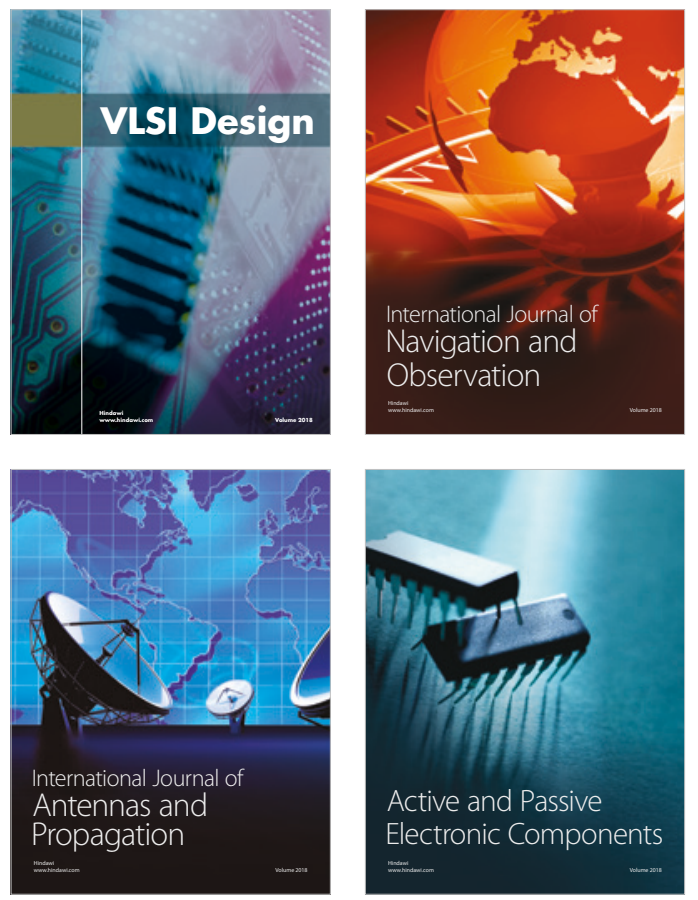
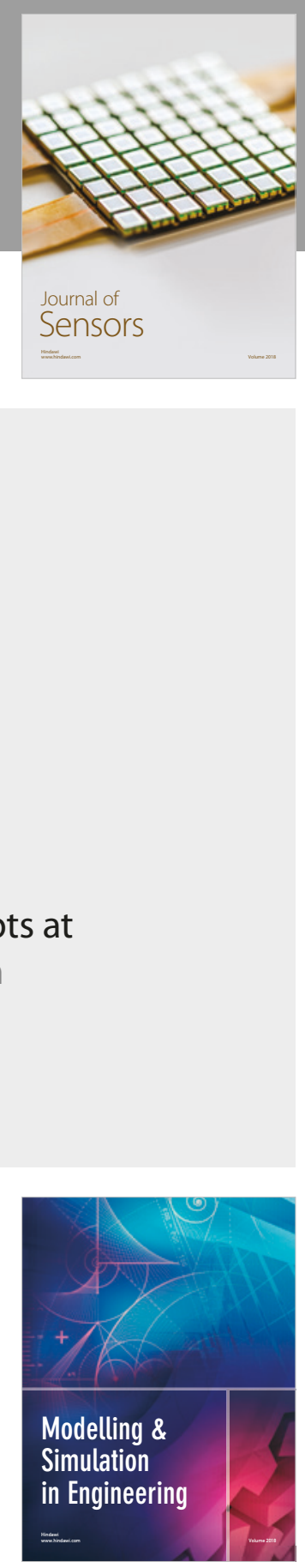

\section{Advances \\ Multimedia}
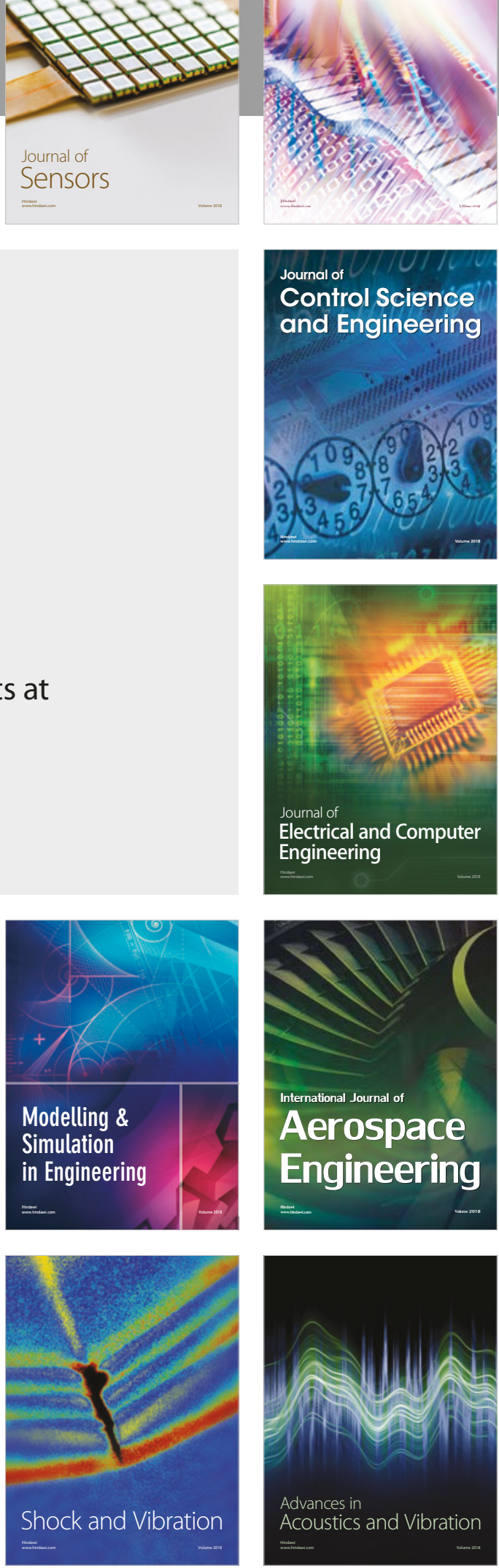